\title{
Comportamiento histórico de los incendios forestales en la provincia de Pinar Del Río, Cuba.
}

\author{
Marcos Pedro Ramos Rodríguez* \\ Ronaldo Viana SoARES**
}

\begin{abstract}
RESUMEN
El conocimiento del comportamiento histórico de los incendios forestales ocurridos en un territorio es de gran utilidad para la planificación eficiente de las medidas de prevención apropiadas para cada territorio, pues permite establecer la tendencia de ocurrencias de los incendios y de sus afectaciones, los períodos del día y del año con mayor riesgo de surgimiento y propagación, las causas del surgimiento, los tipos de especies y los tipos de bosques de acuerdo a su origen, más afectados, a la vez que es posible analizar la efectividad del servicio de protección contra los incendios. El presente trabajo tiene la finalidad de analizar el comportamiento histórico de los incendios forestales en la provincia de Pinar del Río durante el período de 1975 a 1996 con el fin de contribuir al aumento de la efectividad de la prevención contra estos fenómenos, para lo que se utilizó la Base de Datos sobre Incendios Forestales de la Provincia de Pinar del Río a la que se accedió con el Sistema Integrado para el Manejo de Base de Datos sobre Incendios Forestales (SIMBDIF). Entre otros resultados se pueden mencionar que en los bosques de Pinus spp, Eucalyptus spp y Casuarina spp ocurrió el 93,28 \% de los incendios y a ellos correspondió el $94,44 \%$ de las afectaciones. La mayor causa de surgimiento fue el rayo, con un porcentaje del 47,46\%. El 83,69\% de los incendios y el 92,41\% de las afectaciones se presentaron de Marzo a Agosto, ocurriendo desde las 13:00 y hasta las 16:00 horas el 69,43\% de los incendios. Se obtuvo diferencia significativa tanto para las medias del número de incendios ocurridos en bosques naturales y plantados como para las medias del número de hectáreas afectadas en los mismos.
\end{abstract}

* Profesor de la Facultad de Agronomía y Forestal, Universidad de Pinar del Río, Cuba

** Profesor del Curso de Ingeniería Forestal, Universidad Federal del Paraná, Brasil 


\begin{abstract}
The knowledge of the forest fires historical behavior in a territory is of great importance for the efficient planning of the appropriate prevention measures, since it permits to establish the occurrence trend of the fires and the burned areas, the periods of time (days and months) with greater occurrence an propagation risks, the cause of the fires, and the amount of fires and respective burned areas for each vegetation type. This paper relates the historical behavior of the forest fires in the Pinar del Río province from 1975 to 1996 in order to contribute for the increase of efficiency in forest fire prevention. The data base of forest fires in Pinar del Río, that is part of the Integrated System of the Data Base Managing on Forest Fires (SIMBDIF), was used. Plantations of Pinus spp, Eucalyptus spp, and Casuarina spp registered $93.28 \%$ of the fires and $94.44 \%$ of the burned areas. The main cause of the fires was lightning, with $47.46 \%$ of the occurrences. About $83.69 \%$ of the fires and $92.41 \%$ of the burned area were recorded from March to August, and 69.43\% of the occurrences were registered between 1:30 and 4:00 PM. Significant statistical difference was detected for the number of fires and burned areas between natural and planted forests.
\end{abstract}

\title{
INTRODUCCIÓN
}

El fuego, factor ecológico del medio natural que ha participado en la sucesión de la vegetación desde tiempos inmemoriales en distintas zonas del planeta, continúa hoy día, a las puertas del Siglo XXI, llamando la atención del hombre por constituir la peor calamidad que perturba el equilibrio alcanzado por la naturaleza en los ecosistemas forestales, al afectarlos en distinto grado todos los años alcanzándose las cifras a nivel mundial según CALABRI (1991) de más de 10 millones de hectáreas de montes y otras superficies boscosas, lo que representa el 0,2 o el 0,3 por ciento de la superficie total cubierta de vegetación boscosa, observándose un fuerte aumento en el número de incendios y hectáreas afectadas.

La quema de la vegetación trae efectos negativos sobre la estabilidad de los nutrientes, la flora y la fauna, la textura del suelo, la estabilidad ecológica y la temperatura del suelo, todo lo cual causa un impacto ambiental desfavorable, llegando a participar en los Cambios Climáticos Globales.

Los incendios forestales constituyen, sin duda, un fenómeno global, que en el ámbito de nuestro país contribuye en distinto grado a acentuar los cinco principales problemas ambientales identificados para el mismo según la Estrategia Ambiental Nacional (CITMA, 1997) consistentes en la degradación de los suelos, deterioro del saneamiento y de las condiciones ambientales en 
asentamientos humanos, contaminación de las aguas terrestres y marinas, la deforestación y la pérdida de la diversidad biológica, ocurriendo todos los años una media de 298 incendios que afectan un promedio de 4.208,26 ha, siendo la provincia de Pinar del Río la que ocupa el primer lugar en cuanto al número de incendios, ocurriendo en ella el 21,48 \% de los mismos y el segundo lugar en cuanto a las afectaciones, correspondiéndole en este caso el $14,13 \%$ de las mismas.

Estas afectaciones pueden disminuirse si como resultado del análisis del comportamiento histórico de los incendios forestales en la provincia, se implementa un adecuado plan que permita aumentar la efectividad de la prevención contra estos fenómenos. En este sentido es importante establecer la tendencia del número de incendios y de las superficies afectadas pues esto indica la necesidad de mantener o continuar mejorando los niveles alcanzados en la protección de los bosques. De igual forma es importante conocer su distribución a través del año, la semana y el día para fijar los períodos en que deben extremarse las medidas de prevención. El análisis de las causas, por su parte, permite mejorar la planificación de la prevención, mientras que determinar sobre que vegetación ocurre el mayor número de incendios y las mayores afectaciones posibilita destinar los recursos disponibles a las arreas de vegetación más vulnerable.

Estos y otros elementos del comportamiento histórico de los incendios han sido observados en trabajos desarrollados en distintas regiones del mundo (SOARES, 1988 y 1992, en Brasil; VELEZ, 1990, en España; CANAKCIOGLU, 1990, en Turquía;). También en Cuba se han desarrollado estudios con esta finalidad (OHARRIZ et al, 1990; RAMOS, 1996; RAMOS et al 1998). El presente trabajo tiene la finalidad de analizar el comportamiento histórico de los incendios forestales en la provincia de Pinar del Río durante un período de 22 años (1975 a 1996) con el fin de contribuir al aumento de la efectividad de la prevención contra estos fenómenos, para lo que se utilizó la Base de Datos sobre Incendios Forestales de la Provincia de Pinar del Río (BDIFPR) a la que se accedió con el Sistema Integrado para el Manejo de Base de Datos sobre Incendios Forestales (SIMBDIF).

\section{MATERIAL Y METODOS}

\section{UBICACIÓN DE LA INVESTIGACIÓN}

La investigación se desarrolló en la provincia más occidental de la Isla de Cuba, Pinar del Río, la que tiene una superficie geográfica de $10.901 \mathrm{Km} 2$ (1.090.100 ha) y está ubicada (JAULA 1980) entre los $21^{\circ} 45^{`}$ y $23^{\circ} 01^{`}$ de latitud norte, localizados estos lugares en el Cabo Francés y Punta Gobernadora, respectivamente; mientras que su longitud geográfica está entre los $82^{\circ} 51^{`}$ y 
$84^{\circ} 57^{`}$ al oeste, o sea, en los puntos conocidos por la desembocadura del río San Juan - Bayate (limite con la provincia Habana) y el Cabo de San Antonio, respectivamente.

En la provincia la actividad forestal es administrada y dirigida por ocho Empresas Forestales Integrales (EFI) (Figura 1), siendo la de mayor superficie geográfica cubierta de bosques en el país, alcanzando el área perteneciente a las EFI en 1996 un 32,43 \% (353.485,2 ha) del territorio, lo que presupone la importancia de prestar esmerada atención al cuidado de sus bosques.

En el período objeto de análisis el promedio anual de las precipitaciones osciló entre 1.088,88 mm y 2.229,67 mm, observándose durante el año un período lluvioso (Mayo - Octubre) en el que se presenta un aumento de la actividad eléctrica y uno poco lluvioso (Noviembre - Abril), mientras que el promedio de la velocidad del viento osciló entre $7 \mathrm{~km} / \mathrm{h}$ en Agosto y 12,05 $\mathrm{km} / \mathrm{h}$ en Marzo. La temperatura osciló a través del año entre los $21,59^{\circ} \mathrm{C}$ en Enero y los $26,85{ }^{\circ} \mathrm{C}$ en Agosto, oscilando la humedad relativa entre un $76 \%$ en Abril y un $84 \%$ en Septiembre (Tabla 1).

Tabla 1 - Datos meteorológicos de la región de Pinar del Río (promedio mensual para el período de 1975 a 1996)

Table 1 - Meteorological data from the Pinar del Río region (monthly mean for the 1975 to 1996 period)

\begin{tabular}{lcccc}
\hline \multicolumn{1}{c}{ MESES } & PRECIPITACIÓN & TEMPERATURA & $\begin{array}{c}\text { VEL. } \\
\text { VIENTO } \\
(\mathrm{km} / \mathrm{h})\end{array}$ & $\begin{array}{c}\text { HUMEDAD } \\
\text { RELATIVA } \\
(\%)\end{array}$ \\
\hline Enero & $(\mathrm{mm})$ & $\left({ }^{\circ} \mathrm{C}\right)$ & 9,51 & 80 \\
Febrero & 63,77 & 21,59 & 9,84 & 78 \\
Marzo & 60,24 & 21,85 & 12,05 & 77 \\
Abril & 58,52 & 23,22 & 11,31 & 76 \\
Mayo & 76,11 & 24,54 & 9,02 & 80 \\
Junio & 160,30 & 25,89 & 8,46 & 83 \\
Julio & 231,64 & 26,69 & 7,16 & 82 \\
Agosto & 160,54 & 26,52 & 7,00 & 83 \\
Septiembre & 177,72 & 26,85 & 7,17 & 84 \\
Octubre & 217,31 & 26,30 & 8,41 & 83 \\
Noviembre & 151,70 & 25,30 & 9,54 & 82 \\
Diciembre & 81,89 & 23,76 & 8,84 & 81 \\
\hline Total/Promedio & 44,79 & 21,85 & 9,02 & 81 \\
\hline
\end{tabular}


Figura 1 - Empresas forestales integrales de la provincia de Pinar del Río

Figure 1 - Integrated forest enterprises in Pinar del Río province

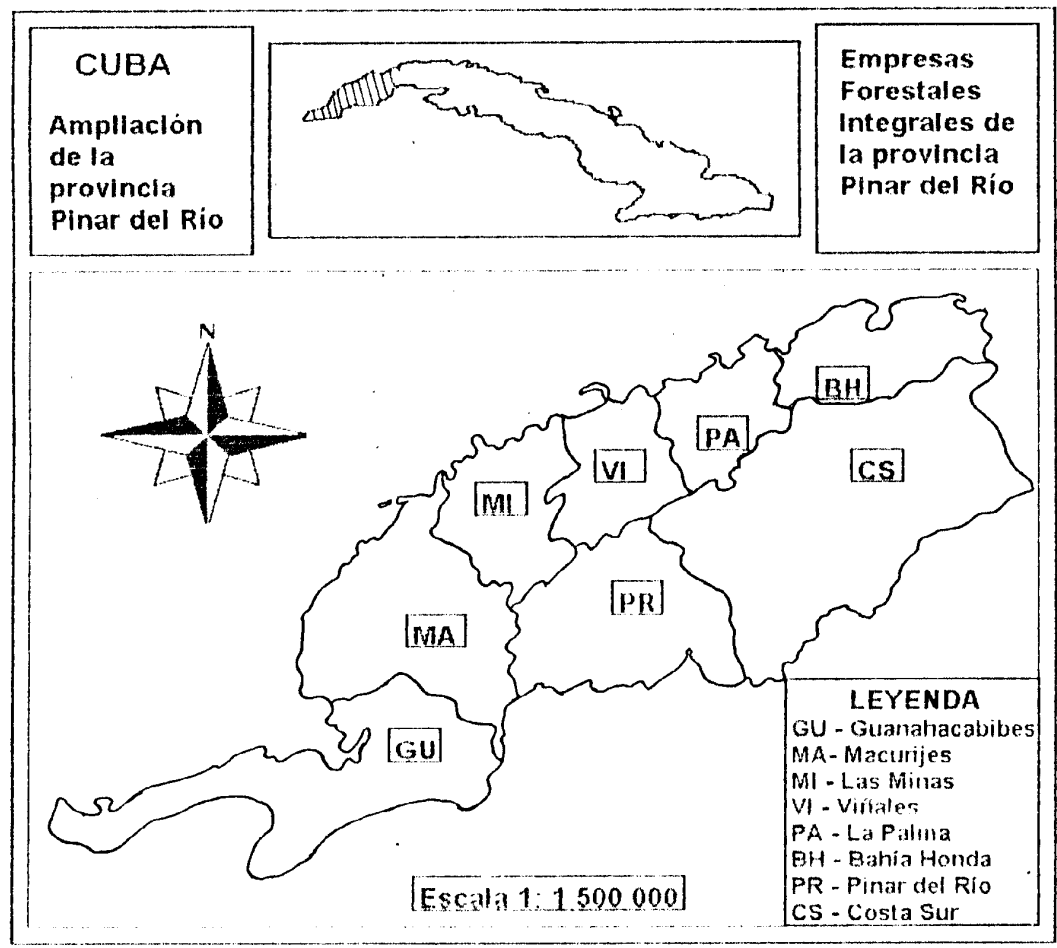

Se ha observado también que desde Noviembre y hasta Mayo se presentan los valores más altos de las velocidades del viento, lo que coincide hasta Abril con el período poco lluvioso y hasta Marzo con los menores valores de las temperaturas mientras que desde Enero y hasta Mayo se presentan los menores valores de la humedad relativa.

Durante el día los menores valores de la humedad relativa se presentan desde las 10:00 horas y hasta las 19:00 horas tanto en Febrero (invierno) como en Junio (verano), período que coincide con el de los valores más altos de las temperaturas.

Las cotas de los lugares donde se desarrollan actividades forestales importantes en la provincia oscilan entre 120 y 250 m.s.n.m. por lo que este factor no determina cambios apreciables de la temperatura entre los distintos lugares del territorio que puedan determinar diferencias importantes en cuanto al secado del material combustible, ocurriendo de forma similar con respecto a 
la exposición, pues además de no existir elevaciones significativas, su orientación ENE - WSW determina que los lados que dan al Sur reciban el sol de la primera mitad del día y los que dan al Norte lo reciban durante la segunda mitad, recibiendo por este motivo ambos lados una radiación similar.

\section{COMPORTAMIENTO HISTÓRICO DE LOS INCENDIOS FORESTALES EN LA PROVINCIA DE PINAR DEL RÍO}

El análisis del comportamiento histórico de los incendios forestales en la provincia se ha basado en las informaciones disponibles en la Base de Datos sobre Incendios Forestales de la provincia de Pinar del Río (BDIFPR) a la que se accedió con el empleo del Sistema Integrado para el Manejo de Bases de Datos sobre Incendios Forestales (SIMBDIF), diseñados por RAMOS et al (1998), abarcándose un período de 22 años (1975 - 1996). El número total de ocurrencias fue de 1.285 incendios, de los cuales 1.220 tenían informaciones suficientes para los analisis presentados en este trabajo.

- Frecuencias y tendencias de incendios y afectaciones.

Como parte del análisis se han determinado las frecuencias del número de incendios y del número de hectáreas afectadas, así como la tendencia de estas variables para lo que se obtuvieron las respectivas ecuaciones de regresión utilizando para ambos casos como variable independiente a los años y como variables dependientes al número de incendios en el primer caso y al número de hectáreas afectadas en el segundo.

- Distribución de los incendios y las afectaciones a través de diferentes períodos de tiempo.

En este caso se obtuvo la distribución mensual del número de incendios y del número de hectáreas afectadas, así como la distribución del número de incendios a través de la semana y a través del día. Para el caso de la distribución semanal del número de incendios se utilizó la prueba no paramétrica de Kruskal - Wallis siguiendo a SIGARROA (1985) con el fin de determinar la existencia o no de diferencia entre los resultados obtenidos para cada uno de los días.

- Distribución de los incendios y las afectaciones a través de la vegetación forestal.

El análisis tuvo el fin de establecer la posible diferencia entre la cantidad de incendios y de hectáreas afectadas en bosques naturales y en plantaciones, para lo que se aplicó una prueba de hipótesis para las medias obtenidas para cada una de estas variables utilizándose un nivel de significación del 0,05. También se obtuvieron los valores correspondientes a estas variables para los cuatro tipos de especies establecidos. A los valores registrados para cada especie durante los 22 años del análisis se les aplicó la prueba no paramétrica de Kruscal - Wallis y posteriormente la prueba de comparaciones múltiples no 
paramétricas para establecer la existencia de posibles diferencias entre los valores obtenidos.

- Distribución de los incendios y las afectaciones a través de las causas.

Se obtuvieron los valores correspondientes al número de incendios y

de afectaciones según las causas que los originan.

- Efectividad del servicio de protección contra incendios.

La efectividad de este servicio se evaluó según los indicadores siguientes:

a) Densidad de incendios: es la cantidad de incendios ocurridos por cada 1000 ha de bosques por año.

b) Densidad de afectaciones: se refiere a la cantidad de hectáreas dañadas por cada 1000 ha de bosques por año.

c) Superficie de bosque dañada por incendio: es el promedio de hectáreas dañadas por cada incendio ocurrido.

d) Porcentaje de la superficie boscosa que se quema por año: es expresado como la superficie quemada entre la superficie cubierta de bosque por 100 .

- Valoración económica de los resultados.

Para la valoración económica se consideró el promedio de hectáreas afectadas cada año en la provincia, se utilizó un valor de las pérdidas para cada hectárea quemada de $\$ 2.100,00$ pesos (OHARRIZ 1991) y que con los resultados propuestos es posible reducir las afectaciones al menos en un $5 \%$ cada año.

\section{RESULTADOS}

\section{COMPORTAMIENTO HISTÓRICO DE LOS INCENDIOS FORESTALES EN PINAR DEL RÍO.}

- Frecuencias y tendencias de incendios y afectaciones.

La frecuencia de incendios durante el período 1975 - 1996 fue de 55,45 ocurrencias por año y el promedio anual de hectáreas afectadas de 460,46 ha. En la Tabla 2 se muestra la distribución de los incendios y las afectaciones a través del período analizado. Con estos datos se analizó la tendencia del número de incendios y de las afectaciones. Las Figuras 2 y 3 muestran que la tendencia de las dos variables es al aumento, lo que es aun más notable en el caso del número de incendios. Este resultado constituye una alerta en cuanto a la necesidad de continuar perfeccionando los sistemas de prevención, detección y extinción utilizados. 
Tabla 2 - Distribución de los incendios y afectaciones en Pinar del Río (1975 a 1996)

Table 2 - Fires and affectations in Pinar del Río (1975 to 1996).

\begin{tabular}{crrrr}
\hline ANOS & \multicolumn{2}{c}{ INCENDIOS } & \multicolumn{2}{c}{ AFECTACIONES } \\
\cline { 2 - 5 }$\left(\mathrm{N}^{\circ}\right)$ & $(\%)$ & $($ ha $)$ & $(\%)$ \\
\hline 1975 & 60 & 4,92 & 880,57 & 8,69 \\
1976 & 28 & 2,30 & 344,75 & 2,40 \\
1977 & 33 & 2,70 & 287,50 & 0,89 \\
1978 & 18 & 1,48 & 90,22 & 2,92 \\
1979 & 67 & 5,49 & 295,48 & 2,11 \\
1980 & 54 & 4,43 & 213,90 & 7,68 \\
1981 & 75 & 6,15 & 777,84 & 3,58 \\
1982 & 44 & 3,61 & 362,73 & 0,41 \\
1983 & 19 & 1,56 & 41,95 & 2,07 \\
1984 & 47 & 3,85 & 209,66 & 12,15 \\
1985 & 66 & 5,41 & 1231,07 & 4,80 \\
1986 & 63 & 5,16 & 496,09 & 3,75 \\
1987 & 60 & 4,92 & 379,80 & 5,09 \\
1988 & 62 & 5,08 & 515,13 & 6,29 \\
1989 & 101 & 8,28 & 637,64 & 4,89 \\
1990 & 68 & 5,57 & 495,72 & 6,68 \\
1991 & 60 & 4,92 & 677,06 & 2,85 \\
1992 & 52 & 4,26 & 288,50 & 3,83 \\
1993 & 68 & 5,57 & 388,32 & 6,53 \\
1994 & 67 & 5,49 & 661,15 & 5,27 \\
1995 & 51 & 4,18 & 553,69 & 3,27 \\
1996 & 57 & 4,67 & 331,42 & 100,00 \\
\hline Totales & 1220 & 100,00 & 10130,19 &
\end{tabular}

- Distribución de los incendios y las afectaciones a través de diferentes períodos de tiempo.

Los valores de la Tabla 3, muestran la distribución mensual de los incendios y las afectaciones de 1975 a 1996, observándose un período de mayores ocurrencias y afectaciones desde Marzo hasta Agosto con el 83,69 \% (1021 incendios) y el 92,41\% (9360,93 ha), respectivamente. 
Figura 2 - Distribución y tendencia de los incendios de 1975 a 1996 en la provincia de Pinar del Río

Figure 2 - Distribution and tendency of fires from 1975 to 1996 in the Pinar del Río province

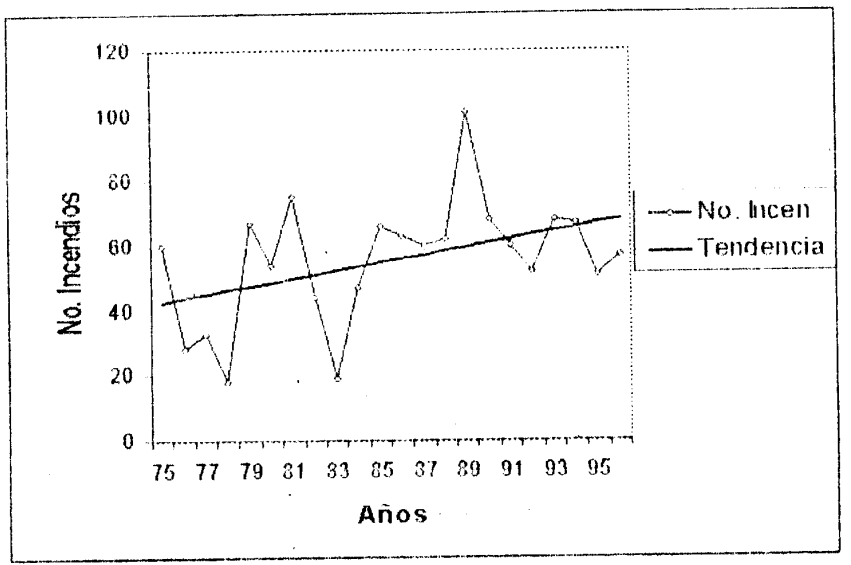

Figura 3 - Distribución y tendencia de las afectaciones de 1975 a 1996 en la provincia de Pinar del Río.

Figure 3 - Distribution and tendency of affectations from 1975 to 1996 in the Pinar del Río province

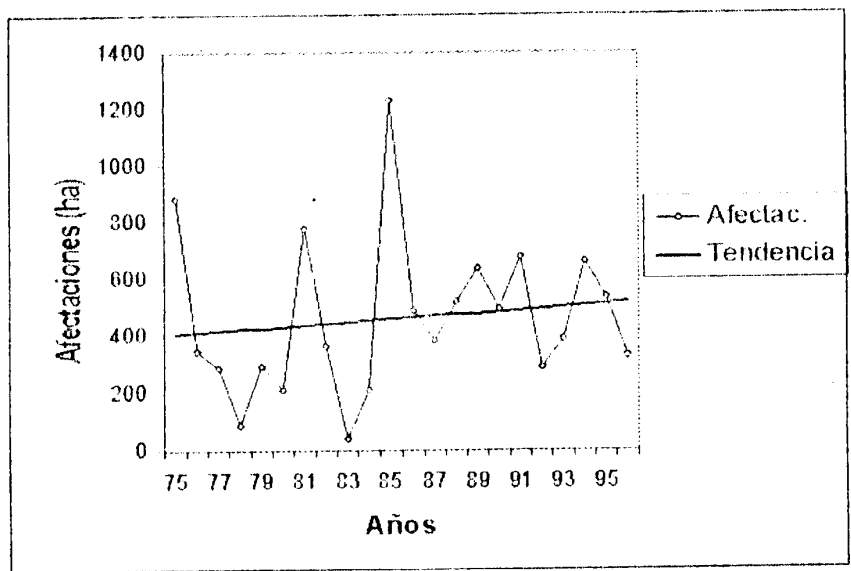


Tabla 3 - Distribución de los incendios y las afectaciones a través del año en el período de 1975 a 1996

Table 3 - Distribución de los incendios y las afectaciones a través del año en el período de 1975 a 1996

\begin{tabular}{lrrrr}
\hline \multicolumn{1}{c}{ MESES } & \multicolumn{2}{c}{ INCENDIOS } & \multicolumn{2}{c}{ AFECTACIONES } \\
\cline { 2 - 5 } & \multicolumn{1}{c}{$\mathrm{N}^{\mathrm{r}}$} & $(\%)$ & $(\mathrm{ha})$ & $(\%)$ \\
\hline Enero & 37 & 3,03 & 112,26 & 1,11 \\
Febrero & 82 & 6,72 & 381,33 & 3,76 \\
Marzo & 163 & 13,36 & 2174,77 & 21,47 \\
Abril & 226 & 18,52 & 2693,25 & 26,59 \\
Mayo & 172 & 14,10 & 1997,69 & 19,72 \\
Junio & 144 & 11,80 & 727,93 & 7,19 \\
Julio & 195 & 15,89 & 1152,70 & 11,38 \\
Agosto & 121 & 9,92 & 614,59 & 6,07 \\
Septiembre & 47 & 3,85 & 143,65 & 1,42 \\
Octubre & 9 & 0,74 & 59,51 & 0,59 \\
Noviembre & 8 & 0,66 & 16,80 & 0,17 \\
Diciembre & 16 & 1,31 & 55,71 & 0,55 \\
\hline \multicolumn{1}{c}{ Totales } & 1220 & 100,00 & 10130,19 & 100,00 \\
\hline
\end{tabular}

En la Tabla 4 se refleja el comportamiento de los incendios durante la semana, correspondiendo al Miércoles el valor más alto con el 16,64 \% (203 incendios), seguido del Jueves con el 16,07 \% (196 incendios). Los menores valores se presentan los Domingos y los Sábados con el 10,98 \% (134 incendios) y el 13,03\% (159 incendios) respectivamente. Esto puede deberse a que durante los días laborables de la semana se incrementa el acceso de personas al bosque, con motivo del desarrollo del proceso de producción forestal. No obstante, no se detectó diferencia significativa para los valores del número de incendios registrados cada día de la semana lo que indica la necesidad de mantener la vigilancia todos los días por igual.

Tabla 4 - Distribución de los incendios a través de la semana en el período de 1975 a 1996

Table 4 - Distribución de los incendios a través de la semana en el período de 1975 a 1996

\begin{tabular}{lcc}
\hline \multirow{2}{*}{ DIAS } & \multicolumn{2}{c}{ INCENDIOS } \\
\cline { 2 - 3 } & $\left(\mathrm{N}^{\mathrm{o}}\right)$ & $(\%)$ \\
\hline Domingo & 134 & 10,98 \\
Lunes & 189 & 15,49 \\
Martes & 175 & 14,35 \\
Miércoles & 203 & 16,64 \\
Jueves & 196 & 16,07 \\
Viernes & 164 & 13,44 \\
Sábado & 159 & 13,03 \\
\hline \multicolumn{1}{c}{ Totales } & 1220 & 100,00 \\
\hline
\end{tabular}


Los valores del comportamiento de las ocurrencias de los incendios durante las distintas horas del día se presentan en la Tabla 5. Se observa que el mayor número ocurre desde las 13:00 y hasta las 16:00 horas, período en el que se registraron $69,43 \%$ de los incendios, alcanzándose el valor más alto con el 21,48 \% (262 incendios) a las 15:00 horas. Durante las primeras horas de la mañana y las últimas de la tarde disminuyen las ocurrencias y por la noche no se han reportado incendios. Este comportamiento está relacionado con el que muestran la temperatura y la humedad relativa durante el día.

Tabla 5 - Distribución de los incendios a través de las horas del día en el período de 1975 a 1996

Table 5 - Distribución de los incendios a través de las horas del día en el período de 1975 a 1996

\begin{tabular}{|c|c|c|c|c|c|}
\hline \multirow[t]{2}{*}{ HORAS } & \multicolumn{2}{|c|}{ INCENDIOS } & \multirow[t]{2}{*}{ HORAS } & \multicolumn{2}{|c|}{ INCENDIOS } \\
\hline & $\left(\mathrm{N}^{0}\right)$ & $(\%)$ & & $\left(\mathrm{N}^{\circ}\right)$ & $(\%)$ \\
\hline $6: 00$ & 1 & 0,08 & $15: 00$ & 262 & 21,48 \\
\hline 7:00 & 5 & 0,41 & $16: 00$ & 219 & 17,95 \\
\hline $8: 00$ & 5 & 0,41 & $17: 00$ & 86 & 7,05 \\
\hline 9:00 & 19 & 1,56 & $18: 00$ & 38 & 3,11 \\
\hline $10: 00$ & 34 & 2,79 & 19:00 & 17 & 1,39 \\
\hline $11: 00$ & 68 & 5,57 & $20: 00$ & 5 & 0,41 \\
\hline 12:00 & 90 & 7,38 & $21: 00$ & 2 & 0,16 \\
\hline 13:00 & 153 & 12,54 & 22:00 & 3 & 0,25 \\
\hline $14: 00$ & 213 & 17,46 & Totales & 1220 & 100,00 \\
\hline
\end{tabular}

- Distribución de los incendios y las afectaciones a través de la vegetación forestal.

En la Tabla 6 se exponen los valores de los incendios ocurridos y las hectáreas afectadas durante los 22 años del estudio en bosques naturales y en plantaciones. Al aplicar la prueba de hipótesis para las medias se detectó diferencia significativa entre las medias de los incendios ocurridos en bosques naturales y en las plantaciones al igual que para el caso de las medias de las hectáreas afectadas en cada tipo de bosque. Se notan incrementos de los valores promedios para el número de incendios y de hectáreas afectadas en los bosques plantados, lo cual coincide con el criterio de diferentes autores que plantean que el bosque natural, mixto y disetáneo en la medida en que se aproxima a su estado clímax, presenta menores posibilidades para el surgimiento y propagación de los incendios, por lo que se debe prestar esmerada atención a la protección contra los incendios en las plantaciones. 
Tabla 6 - Distribución de los incendios y las afectaciones según el tipo de bosque en el período de 1975 a 1996

Table 6 - Distribución de los incendios y las afectaciones según el tipo de bosque en el período de 1975 a 1996

\begin{tabular}{lcccc}
\hline \multirow{2}{*}{ TIPO DE BOSQUE } & \multicolumn{2}{c}{ INCENDIOS } & \multicolumn{2}{c}{ AFECTACIONES } \\
\cline { 2 - 5 } & \multicolumn{1}{c}{$\left(\mathrm{N}^{\circ}\right)$} & $(\%)$ & $($ ha) & $(\%)$ \\
\hline Natural & 491 & 38,21 & 3686,86 & 36,39 \\
Plantación & 794 & 61,79 & 6443,33 & 63,61 \\
\hline Totales & 1285 & 100,00 & 10130,19 & 100,00 \\
\hline
\end{tabular}

En la Tabla 7 se reflejan los valores de los incendios iniciados y las afectaciones por ellos producidas en las áreas ocupadas por los distintos tipos de especies. Cabe destacar que de los cerca de 350.000 ha de bosques en la Provincia de Pinar del Rio, aproximadamente $34,1 \%$ corresponden a bosques de Pinus spp, 2,8\% a Eucalyptus spp, 0,5\% a Casuarina spp y 62,6\% a otras latifoliadas. El 76,72 \% (936 incendios) se originaron en Pinus spp. Siguiéndole en orden descendente los originados en Eucalyptus spp con el $11,56 \%$ (141), en otras latifolias con el 6,72\% (82) y en Casuarina spp con el $5,00 \%$ (61). Se obtuvo diferencia significativa entre los valores mostrados por cada especie. La prueba de comparaciones múltiples no paramétricas permitió establecer que solo no existe diferencia entre el número de incendios registrados en el Eucalyptus spp y otras latifolias.

También la mayor cantidad de afectaciones se produjo en el Pinus spp al que corresponde el 66,74\% (7660,62 ha) de las mismas, seguido por el Eucalyptus spp con el 21,74 \% (2201,90 ha), la Casuarina spp con el 5,96\% (603,37 ha) y otras latifolias con un 5,57 \% (564,30 ha). En este caso también se obtuvo diferencia significativa entre las especies afectadas. La prueba de comparaciones múltiples no paramétricas dio por resultado que solo entre los valores obtenidos para la Casuarina spp y otras latifolias no existe diferencia significativa. Así se pone de manifiesto la importancia que debe prestarse a la protección contra los incendios en las áreas ocupadas por Pinus spp, el Eucalyptus spp y Casuarina spp, haciéndose necesario exigir, a los niveles correspondientes, tener en cuenta debidamente en los proyectos de reforestación la protección contra los incendios. 
Tabla 7 - Distribución de los incendios y las afectaciones según las especies en el período de 1975 a 1996

Table 7 - Distribución de los incendios y las afectaciones según las especies en el período de 1975 a 1996

\begin{tabular}{lrrrr}
\hline \multirow{2}{*}{ ESPECIES } & \multicolumn{2}{c}{ INCENDIOS } & \multicolumn{2}{c}{ AFECTACIONES } \\
\cline { 2 - 5 } & \multicolumn{1}{c}{$\left(\mathrm{N}^{\circ}\right)$} & $(\%)$ & $(\mathrm{ha})$ & $(\%)$ \\
\hline Pinus spp & 936 & 76,72 & 6760,62 & 66,74 \\
Eucalyptus spp & 141 & 11,56 & 2201,90 & 21,74 \\
Casuarina spp & 61 & 5,00 & 603,37 & 5,96 \\
Otras latifolias & 82 & 6,72 & 564,30 & 5,56 \\
\hline \multicolumn{1}{c}{ Totales } & 1220 & 100,00 & 10130,19 & 100,00 \\
\hline
\end{tabular}

- Distribución de los incendios y las afectaciones a través de las causas.

Los valores correspondientes al número de incendios ocurridos según cada causa y las hectáreas afectadas por estos se presentan en la Tabla 8. Se observa que el rayo es la principal causa de surgimiento, correspondiendo a ella el 47,46 $\%$ (579) del total, siguiéndole las negligencias, las desconocidas y la intencional con el 27,38 \% (334); 22,62 \% (276) y $2,54 \quad \% \quad$ (31) respectivamente, mientras que para las afectaciones son los incendios originados por negligencias los que más áreas han afectado con un 39,42\% (3992,99 ha).

Tabla 8 - Distribución de los incendios y las afectaciones según las causas, en el período de 1975 a 1996

Table 8 - Distribución de los incendios y las afectaciones según las causas, en el período de 1975 a 1996

\begin{tabular}{lrrrr}
\hline \multirow{2}{*}{ CAUSAS } & \multicolumn{2}{c}{ INCENDIOS } & \multicolumn{2}{c}{ AFECTACIONES } \\
\cline { 2 - 5 } & \multicolumn{1}{c}{$\left(\mathrm{N}^{\circ}\right)$} & $(\%)$ & $($ ha $)$ & $(\%)$ \\
\hline Rayos & 579 & 47,46 & 2877,39 & 28,40 \\
Negligencias & 334 & 27,38 & 3992,99 & 39,42 \\
Intencionales & 31 & 2,54 & 703,00 & 6,94 \\
Desconocidas & 276 & 22,62 & 2556,81 & 25,24 \\
\hline \multicolumn{1}{c}{ Totales } & 1220 & 100,00 & 10130,19 & 100,00 \\
\hline
\end{tabular}

- Efectividad del servicio de protección contra incendios.

En la Tabla 9 se muestran los valores de la densidad de incendios, densidad de afectaciones, el promedio de hectáreas afectadas por incendios y el porcentaje que se afecta de la superficie cubierta de bosques de 1989 a 1996. Si se comparan estos valores con los encontrados en la literatura internacional (SOARES 1992), se puede comprobar que los resultados son comparables a los encontrados en países de gran eficiencia en control de incendios forestales. 
Tabla 9 - Densidad de incendios (D.I.), densidad de afectaciones (D.A.), promedio de hectáreas quemadas por incendio (H.I.) y porcentaje de la superficie total cubierta afectada (P.A.) en Pinar del Río

Table 9 - Densidad de incendios (D.I.), densidad de afectaciones (D.A.), promedio de hectáreas quemadas por incendio (H.I.) y porcentaje de la superficie total cubierta afectada (P.A.) en Pinar del Río

\begin{tabular}{lllrc}
\hline \multicolumn{1}{c}{ AÑOS } & D.I. & D.A. & H.I. & P.A. \\
\hline 1989 & 0,28 & 1,79 & 6,31 & 0,179 \\
1990 & 0,19 & 1,40 & 7,29 & 0,140 \\
1991 & 0,17 & 1,90 & 11,28 & 0,190 \\
1992 & 0,15 & 0,81 & 5,55 & 0,081 \\
1993 & 0,19 & 1,09 & 5,71 & 0,109 \\
1994 & 0,14 & 1,86 & 9,87 & 0,186 \\
1995 & 0,14 & 1,50 & 10,46 & 0,150 \\
1996 & 0,16 & 0,94 & 5,82 & 0,094 \\
\hline Media & 0,18 & 1,41 & 7,79 & 0,141 \\
\hline
\end{tabular}

- Valoración económica de los resultados

Cada año en la provincia de Pinar del Río se afectan como promedio 460,46 ha por lo que de acuerdo con el epígrafe 2.2.6 debe evitarse la pérdida cada año de aproximadamente $\$ 48.342,00$ pesos.

\section{CONCLUSIONES}

Los resultados obtenidos en este trabajo permiten arribar a las siguientes conclusiones:

1) La frecuencia anual de incendios y de afectaciones fue de 55,45 incendios $y$ de 460,46 ha respectivamente, observándose en ambos casos que la tendencia es al aumento.

2) Las mayores posibilidades de surgimiento y propagación de incendios fue observado de Marzo a Agosto, ocurriendo en ese período el 83,69 \% de los incendios y el $92,41 \%$ de las afectaciones; no se detectaran diferencias significativas en cuanto al número de incendios ocurridos a través de los diferentes días de la semana y desde las 13:00 y hasta las 16:00 horas, cuando ocurre la mayor cantidad de los incendios.

3) Se detectaran diferencias significativas para un nivel del 0,05, tanto para las medias de los incendios ocurridos en bosques naturales y plantados, como para las medias de las hectáreas afectadas en estos tipos de bosques, favoreciendo en ambos casos el incremento a los bosques plantados, mientras que en bosques de Pinus spp, Eucalyptus spp y Casuarina spp se presentaran los mayores porcentajes para el número de incendios y de hectáreas afectadas. 
4) La principal causa de surgimiento de incendio en la provincia fue el rayo, alcanzando el 47,46\%, mientras que en el caso de las afectaciones la principal causa fue la negligencia con un $39,42 \%$.

5) Los indicadores de la efectividad del servicio de protección contra incendios muestran valores similares a los alcanzados por otros países.

\section{BIBLIOGRAFIA CITADA}

CALABRI, G. 1991. Problemas y perspectivas relativas a los incendios forestales, su prevención y su dominio. Actas del $\mathbf{1 0}^{\mathbf{0}}$ Congreso Forestal Mundial. París.

CANAKCIOGLU, H. 1990. Forest Fire in Turkey. Proceedings of Forest Fire Research and Protection from Fire. XIX IUFRO World Congress. Canadá, p. 42 - 48.

CITMA 1997. Estrategia Ambiental Nacional. Ministerio de Ciencia, Tecnología y Medio Ambiente. Cuba. 27 p.

JAULA, J.A. 1980. Caracterización fisiográfica de la provincia de Pinar del Río. Inédito. 10 p.

OHARRIZ, S.; VALDES, C. y LLORENTE, E.B. 1990. Estadística de los incendios forestales en Cuba durante el período 1981 - 1985. Centro de Información y Documentación Agropecuario. Cuba. 40 p.

OHARRIZ, S. 1991. Protección contra incendios forestales Editorial Pueblo y Educación. Cuba. 76 p.

RAMOS, M.P. 1996. Comportamiento de los incendios forestales en la provincia de Pinar del Río de 1975 a 1994. Trabajo presentado en el IV Congreso Internacional sobre Desastres. Cuba.

RAMOS, M.P.; RODRIGUEZ, P.G. Y LEON, L.E. 1998. Sistema Integrado para el Manejo de Bases de Datos sobre Incendios Forestales (SIMBDIF); Guía del Usuario. Cuba, XII Forum de Ciencia y Técnica de la Universidad de Pinar del Río y en el I Taller Nacional de Ingeniería y Arquitectura para la Reducción de los Desastres. 21p.

SIGARROA, A. 1985. Biometría y diseño experimental. Segunda Parte. Editorial Pueblo y Educación, Cuba. 793 p.

SOARES, R.V. 1988. Perfil dos incendios florestais no Brasil, de 1984 a 1987. Revista Floresta 18 (1-2): 94-121. 
SOARES, R.V. 1992. Ocorrência de incendios em povoamentos florestais. Revista Floresta 22 (1-2): 39-54.

VELEZ, R. 1990. Los incendios forestales en el Mediterráneo: perspectiva regional. Revista Unasylva. Vol.41, No 162: 3 - 9. 